\title{
Tetraspanin 1 is involved in survival, proliferation and carcinogenesis of pancreatic cancer
}

\author{
FENG-QIANG HOU, XI-FENG LEI，JIAN-LONG YAO，YI-JIN WANG and WEI ZHANG \\ Department of General Surgery, The Weinan Central Hospital, Weinan, Shaanxi 714000, P.R. China
}

Received June 25, 2015; Accepted July 8, 2015

DOI: $10.3892 /$ or.2015.4272

\begin{abstract}
Pancreatic cancer (PCC) is one of the most difficult cancers to treat and the 10th leading cause of cancer-related death in worldwide. Studies have demonstrated that the tetraspanin 1 (Tspan1) is overexpressed in various cancers and may be a potential therapeutic strategy for the treatment of different cancers. However, the possible role of Tspan1 in PCC is still unknown. In the present study, our data revealed that the increased Tspan1 in PCC tissues was associated with the clinicopathological features and survival rate of PCC patient. We also investigated the effects of Tspan1 gene knockdown on the biological behavior of human PCC. The expression of Tspan1 (detected by immunohistochemistry, qRT-PCR and western blot analysis) derived from human PCC tissues and cell lines (AsPC-1 and PANC-1), were significantly elevated compared with those of the control $(\mathrm{P}<0.05)$. Transfection with siRNA-targeting Tspan 1 significantly decreased proliferation, increased the apoptosis and reduced migration and invasion of AsPC-1 and PANC-1 cells. The present study demonstrated that Tspan1 plays an important role in PCC carcinogenic progression, including migration and invasion. The siRNA targeting of Tspan1 may be a potential therapeutic strategy for the treatment of PCC.
\end{abstract}

\section{Introduction}

Pancreatic cancer (PCC) represents one of the leading causes of cancer-related mortality in industrialized countries (1). Despite surgical resection, radiation, and chemotherapy, >94\% of patients with PCC do not survive beyond 5 years. The poor prognosis of this disease is mainly due to its early systemic metastasis (1-3).

It is well established that the tetraspanin family of four-pass transmembrane proteins has been implicated in fundamental

Correspondence to: Dr Wei Zhang, Department of General Surgery, The Weinan Central Hospital, Weinan, Shaanxi 714000, P.R. China

E-mail: zhangwei_weinan@126.com

Key words: apoptosis, migration, small interference RNA, invasion, pancreatic cancer, tetraspanin 1 , proliferation biological processes, including cell adhesion, migration, and proliferation (4). Tetraspanins interact with various transmembrane proteins, establishing a network of large multimolecular complexes that allows specific lateral secondary interactions (5). In animals, the tetraspanins are a large superfamily of membrane proteins that play important roles in organizing various cell-cell and matrix-cell interactions and signal pathways based on such interactions $(6,7)$.

Tetraspanins are a heterogeneous group of 4-transmembrane proteins that segregate into so-called tetraspanin-enriched microdomains (TEMs) along with other cell surface proteins such as integrins. TEMs of various types are reportedly involved in the regulation of cell growth, migration and invasion of several tumor cell types, both as suppressors or supporting structures (8). Tetraspanin 1 (Tspan1), a member of the transmembrane 4 superfamily of tetraspanins, is overexpressed in high-grade cervical intraepithelial neoplasia (CIN) and terminal carcinomas (8), lung cancer (9), colon cancer (10), breast cancer (11), as well as squamous cell carcinoma (12). However, the precise function of Tspan1 in the context of PCC is not known.

In the present study, quantitative RT-PCR (qRT-PCR) and western blot analysis were employed to explore the expressions of Tspan1 in human PCC tissues, adjacent normal tissues and human AsPC-1 and PANC-1 cell lines. Immunohistochemistry (IHC) was also used to detect the subcellular locations of Tspan1 in human PCC tissues. The corrections between Tspan1 expression in PCC and clinicopathological features were analyzed. Then, virus carrying a small interference RNA (siRNA) targeting the human Tspan1 gene was constructed and transfected into PCC cells. After transfection, Tspan1 expression was detected by qRT-PCR and western blot analysis. The cell proliferation and apoptosis fractions were also evaluated by MTT assay and flow cytometry (FCM). Additionally, the Transwell assays were employed to explore the effects of Tspan1 knockdown on the migration and invasion of PCC cells in vitro.

\section{Materials and methods}

Ethical aspects. This study complied with the International Ethical Declaration and was approved by the Human Ethics Committee and the Research Ethics Committee of Shaanxi Province of China. Through the surgery consent form, patients were informed that the resected specimens were kept by our 
hospital and may be used for scientific research, and that their privacy would be maintained.

Clinical samples collections. Forty-five patients attending the clinic in the Weinan Central Hospital, between February 2013 to December 2014, were invited to participate in the study. The patients ranged in age from 35 to 72 years, with a median age of 51 years. All of them were operated for the first time, without any antitumor treatment. The specimens were histopathologically verified as PCC. Then the tumor samples and matched normal adjacent tissues were obtained, which were taken at least $0.5 \mathrm{~cm}$ distal to tumor margins. The biopsies obtained were divided into two fragments immediately after surgery. One fragment was immediately stored at $-80^{\circ} \mathrm{C}$ until nucleic acid and protein isolation. The remaining fragment was fixed in $4 \%$ formaldehyde for two days and then paraffinembedded. The paraffin blocks were sliced and stained with IHC for histological examination.

IHC staining. Immunohistochemistry was performed to determine the Tspan1 expression in normal adjacent tissue and PCC tissues. Briefly, tumor samples and the matched control tissues were fixed in $4 \%$ formaldehyde, and embedded in paraffin wax. Fixed tumor samples were prepared into 4- $\mu \mathrm{m}$ frozen sections and incubated with $2 \%$ goat serum at $37^{\circ} \mathrm{C}$ for $20 \mathrm{~min}$, followed by incubation with rat anti-human Tspan1 (1:400, sc-376551; Santa Cruz Biotechnology) at $4^{\circ} \mathrm{C}$ overnight. After washing with PBS, the sections were incubated with horseradish peroxidase (HRP)-conjugated rabbit anti-rat $\operatorname{IgG}(\mathrm{HRP}-\mathrm{IgG})$ at $37^{\circ} \mathrm{C}$ for $30 \mathrm{~min}$ and colored with 3,3'-diaminobenzidine (DAB) at room temperature. PBS was substituted for the anti-Tspan1 antibody in negative control subjects. All sections were then blindly analyzed by three experienced pathologists under a light microscope. The clinicopathologic data and patient outcomes were not known by the pathologists. The results of IHC staining were evaluated as described (13). The cases were classified into positive groups by the intensity and proportion of the immunostained cancer cells or Tspan1. The proportion of positive cells was assessed as low (5-25\% cells stained), medium (25-50\% cells stained), and high ( $>75 \%$ cells stained).

Quantitative reverse-transcription PCR. The expression of Tspan1 in the PCC specimens and the cell lines were detected by quantitative real-time PCR (qRT-PCR). Tumor samples (50 mg) were ground under liquid nitrogen, lysed with $1 \mathrm{ml}$ of TRIzol (Takara, Japan), and total RNA was extracted using TRIzol (Invitrogen, Carlsbad, CA, USA). Total RNA $(2 \mu \mathrm{g})$ was added to the tumor extract with Moloney Murine Leukemia Virus Reverse Transcriptase (MMLV-RT; Takara) to synthesize cDNA, and the reverse transcript was used as the template for qRT-PCR using a Tower qRT-PCR system (Analytik, Jena, Germany). The qRT-PCR was conducted using 2X Mix SYBR Green I (10 $\mu$; Biosea, USA), primer $(0.25 \mu \mathrm{l}, 10 \mathrm{pmol} / \mathrm{l})$, template DNA $(1 \mu \mathrm{l})$, and sterile water $(8.5 \mu \mathrm{l})$. All PCR reactions included initial denaturation and multiple cycles at $95^{\circ} \mathrm{C}$ for $3 \mathrm{~min} ; 39$ cycles at $95^{\circ} \mathrm{C}$ for $10 \mathrm{sec}$, $55^{\circ} \mathrm{C}$ for $10 \mathrm{sec}$, and $72^{\circ} \mathrm{C}$ for $30 \mathrm{sec}$; followed by $95^{\circ} \mathrm{C}$ for $10 \mathrm{sec}, 65^{\circ} \mathrm{C}$ for $5 \mathrm{sec}$, and a final $95^{\circ} \mathrm{C}$ for $15 \mathrm{sec}$. The primer for each gene was synthesized by Invitrogen. The real-time
PCR primers used to quantify GAPDH expression were: forward, 5'-CGAGATCCCTCCAAAATCAA-3' and reverse, 5'-TTCACACCCATGACGAACAT-3'; and for Tspan1 were: forward, 5'-TGGGCTGCTATGGTGCTA-3' and reverse, 5'-T-GCAGGTTTCATTGGCTGT-3'. Expression of Tspan1 was normalized to endogenous GAPDH expression.

Western blot analysis. Tspan1 protein levels both in PCC tissues and cell lines were determined by western blot analysis. Briefly, samples were lysed for $30 \mathrm{~min}$ in CytoBuster Protein Extraction Buffer (Novagen, USA) and centrifuged at $12,000 \mathrm{rpm}$. The supernatant was collected, total protein was measured, and $50 \mu \mathrm{g}$ was used for $10 \%$ sodium dodecyl sulfate-polyacrylamide gel electrophoresis (SDS-PAGE). The protein was then transferred to a nitrocellulose (NC) membrane and sealed with Tris-buffered saline Tween-20 (TBST) containing 5\% non-fat milk powder. The membrane was subsequently incubated with goat anti-human Tspan1 proteins and mouse anti-human GAPDH (1:500, sc-81545; Santa Cruz Biotechnology) at $4^{\circ} \mathrm{C}$ overnight. After washing in TBST, the membrane was incubated with HRP-conjugated secondary antibodies $(1: 2,000)$ at $25^{\circ} \mathrm{C}$, and the protein quantity was determined using electrochemiluminescence (ECL) technique (BestBio, USA). The results were photographed using the JS Gel Imaging system and the grey density was calculated using SensiAnsys software (both from Peiqing, China).

Cell culture. Human PCC cell lines AsPC-1 and PANC-1, as well as normal human pancreatic hTERT-HPNE cell lines were all purchased from the Cell Bank of the Chinese Academy of Sciences.

All the above cells were cultured in specific medium supplemented with $10 \%(\mathrm{v} / \mathrm{v})$ fetal bovine serum (FBS) and $1 \%$ antibiotics at $37^{\circ} \mathrm{C}$ in a humidified incubator under $5 \% \mathrm{CO}_{2}$ condition (14).

Tspan1 knockdown. According to the CDS of Tspan1 recorded in neucleopeptide, we predesigned siRNA targeting the human Tspan1 gene (gene ID, 10103) (http://RNAiDesigner.invitrogen.com). The siRNA sequences targeting Tspan1 were as follows: si-1, 5'-CCTCAGCAGTTCCCTCTTT-3'; si-2, 5'-GCC TTGGTGTAC-ACCACAA-3'; and si-3, 5'-GCCTGCCATC AAGAAAGAT-3'. Lentivirus was packaged in 293T cells using Lipofectamine 2000 (Invitrogen) and virus titers were determined. Target cells, including AsPC-1 and PANC-1 cells, were infected with $1 \times 10^{6}$ recombinant lentivirus-transducing units in the presence of $6 \mu \mathrm{g} / \mathrm{ml}$ polybrene (Sigma), respectively. The efficiency of knockdown was tested by qRT-PCR and western blot analysis. All experiments were performed in triplicate.

MTT assay. Cell viability was determined using the tetrazolium salt 3-(4,5-dimethylthiazol-2-yl)-2,5-diphenyltetrazolium bromide (MTT) assay. Briefly, cells were plated into 96-well culture plates at an optimal density of $5 \times 10^{3}$ cells $/ \mathrm{ml}$ in $200 \mu \mathrm{l}$ of culture medium/well. After 24-96 h of culture, $20 \mu \mathrm{l}$ of $5 \mathrm{mg} / \mathrm{ml} \mathrm{MTT}$ was added to each well and incubated at $37^{\circ} \mathrm{C}$ for $4 \mathrm{~h}$. The medium was then gently aspirated and $150 \mu \mathrm{l}$ of dimethyl sulfoxide (DMSO) was added to each well to solubilize the formazan crystals. The optical density of each 
Table I. Correlations between Tspan1 expression in PCC and clinicopathological features.

\begin{tabular}{|c|c|c|c|c|c|c|}
\hline & \multicolumn{4}{|c|}{ Tspan1 expression } & \multirow[b]{2}{*}{$\mathrm{r}_{\mathrm{s}}$} & \multirow[b]{2}{*}{ P-values } \\
\hline & Negative & Low & Medium & High & & \\
\hline \multicolumn{7}{|l|}{ Gender } \\
\hline Male & 4 & 8 & 5 & 8 & 0.127 & 0.61 \\
\hline Female & 3 & 5 & 7 & 6 & 0.119 & 0.59 \\
\hline Tumor size $(\mathrm{cm})$ & $2.208 \pm 0.25$ & $3.109 \pm 1.13$ & $4.027 \pm 1.98$ & $5.924 \pm 1.72$ & 9.561 & 0.000326 \\
\hline Histological grade & & & & & 0.103 & 0.34 \\
\hline $\mathrm{G} 1$ & 5 & 3 & 4 & 5 & & \\
\hline $\mathrm{G} 2$ & 1 & 6 & 3 & 4 & & \\
\hline G3 & 1 & 4 & 5 & 5 & & \\
\hline LN metastasis & & & & & 0.311 & 0.003 \\
\hline NO & 6 & 9 & 4 & 4 & & \\
\hline $\mathrm{N} 1$ & 1 & 3 & 8 & 10 & & \\
\hline pTNM stage & & & & & 0.295 & 0.007 \\
\hline $\mathrm{I}, \mathrm{II}$ & 6 & 7 & 2 & 2 & & \\
\hline III & 1 & 3 & 4 & 5 & & \\
\hline IV & 0 & 2 & 6 & 7 & & \\
\hline
\end{tabular}

Tumor size $(\mathrm{cm})$ : Expressed as the mean. $\mathrm{F}=9.0125, \mathrm{P}<0.05$ (Anova analysis); p-values were calculated by Spearman's rank-correlation test ( $\mathrm{n}=45)$; Tspan1, tetraspanin 1; PCC, pancreatic cancer; pTNM, pathological tumor node metastasis.

sample was immediately measured using a microplate reader (Bio-Rad, Hercules, CA, USA) at $490 \mathrm{~nm}$.

Apoptosis assay. A propidium iodide (PI) and Annexin VFITC flow cytometry assay (BD Pharmingen) was used to detect the apoptosis rate in the cells after Tspan1 transfection. Briefly, $1 \times 10^{6}$ cells/well were cultured in 6 -well plates in the absence of $10 \%$ FBS for $48 \mathrm{~h}$. Adherent cells were detached with $0.25 \%$ trypsin without EDTA in $1 \mathrm{X}$ PBS. Cells were harvested in complete RPMI-1640 medium and centrifuged at $1,000 \mathrm{rpm}$ for $5 \mathrm{~min}$. Each of the cell lines was washed with 1X PBS and stained with $50 \mu \mathrm{g} / \mathrm{ml}$ PI and Annexin V-FITC, following the manufacturer's instructions.

Cell migration and invasion assay. We employed BioCoat Matrigel invasion chambers (BD Biosciences, Bedford, MA, USA) to compare the effect of Tspan1 knockdown on in vitro invasion of AsPC-1 and PANC-1 cells as previously described (15). Briefly, for the invasion assay, Costar Transwell 8- $\mu \mathrm{m}$ inserts were coated with $50 \mu \mathrm{g}$ reduced serum Matrigel (BD Biosciences). Invasion chambers were coated with Matrigel, and $1 \times 10^{6}$ cells were added per chamber. Medium supplemented with $10 \%$ FBS was used in the lower chamber. Following incubation the cells that had invaded through the membrane were fixed and stained before the membrane was removed and mounted on a slide for microscopic assessment. Invasive cells were visualized at x40 magnification and the number of cells in five random fields was counted and an average calculated. For migration assays, the same procedure was used excluding the Matrigel. After $12 \mathrm{~h}$, non-invading cells and media were removed, and cells on the lower surface of the membrane were fixed with polyoxymethylene and stained with $0.1 \%$ crystal violet (both from Sigma) for $0.5 \mathrm{~h}$. Stained cells were counted under a microscope in five randomly selected fields, and the average was used to indicate cell migration and invasion. All experiments were performed in triplicate $(15,16)$.

Statistical analysis. SPSS v11.5 (SPSS Inc., Chicago, IL, USA) was used for statistical analysis. Data are presented as means \pm standard deviation. The unpaired t-test was used for comparison between groups. Association between Tspan1 expression and other clinicopathological factors of the tumor were assessed by the Fisher's exact test (two-sided) for categorical variables and $\chi^{2}$ test were used to compare ordinal variables. The grading-related data were analyzed by Spearman's test. A P $<0.05$ was considered statistically significant.

\section{Results}

Upregulation of Tspanl expression in human PCC tissues. Tspan1 staining in normal adjacent tissue was weak relative to PCC tissues. The IHC positive files of Tspan1 exhibited light yellow to brown staining (Fig. 1).

Either qRT-PCR or western blot analysis showed that the expression of Tspan1 in PCC tissue was significantly stronger than that of normal tissues $(\mathrm{P}<0.05$, Fig. 1$)$.

Spearman's analysis showed that the Tspan1 level was correlated with the lymph node metastasis $(\mathrm{r}=0.311, \mathrm{P}<0.05)$ and the pathological tumor node metastasis (pTNM) stages $(r=0.295, \mathrm{P}<0.05)$ in these 45 cancer samples (Table I). Within a period of 60 Smonths of the follow-up, 15 cancer-related deaths occurred, all of the deaths come from patients with 

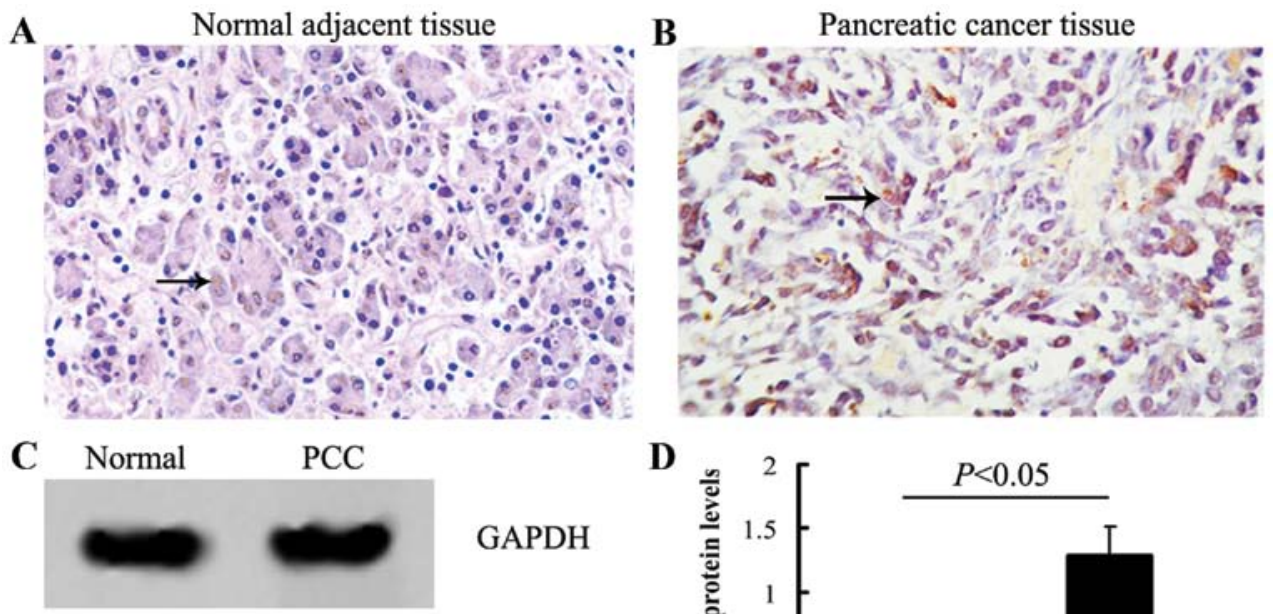

GAPDH

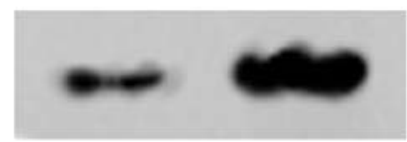

Tspan1

D

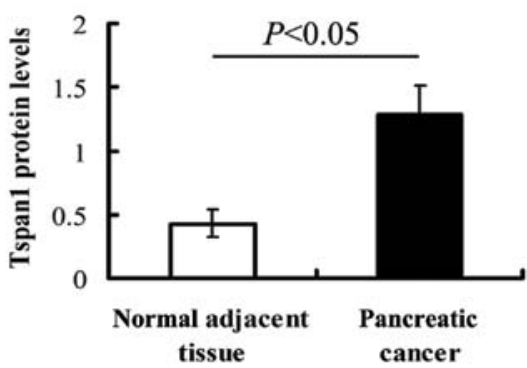

$\mathbf{E}$ $P<0.05$

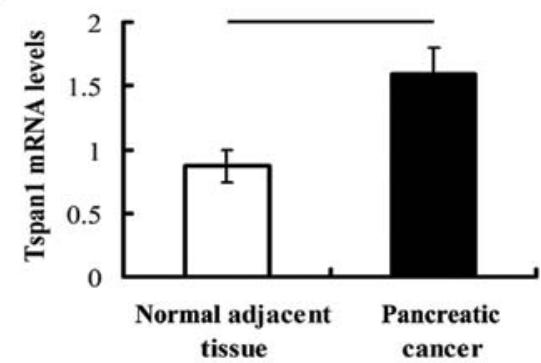

Figure 1. IHC-positive staining of Tspan1 in PCC and normal adjacent tissue. Normal, normal adjacent tissue; PCC, pancreatic cancer tissue (A and B) Representative IHC files of Tspan1 in normal adjacent (A) and PCC tissue (B). There is more intense yellow or yellow-brown staining for Tspan1 in PCC tissue than that in normal adjacent tissue. (C and D) The quantities analysis of protein levels of Tspan1. (E) The quantities analysis of relative mRNA levels of Tspan1. The arrow shows the representative immunostaining of Tspan1. Magnification, $x 200$. Values plotted are means \pm SD ( $n=45)$. Tspan1, tetraspanin 1 .

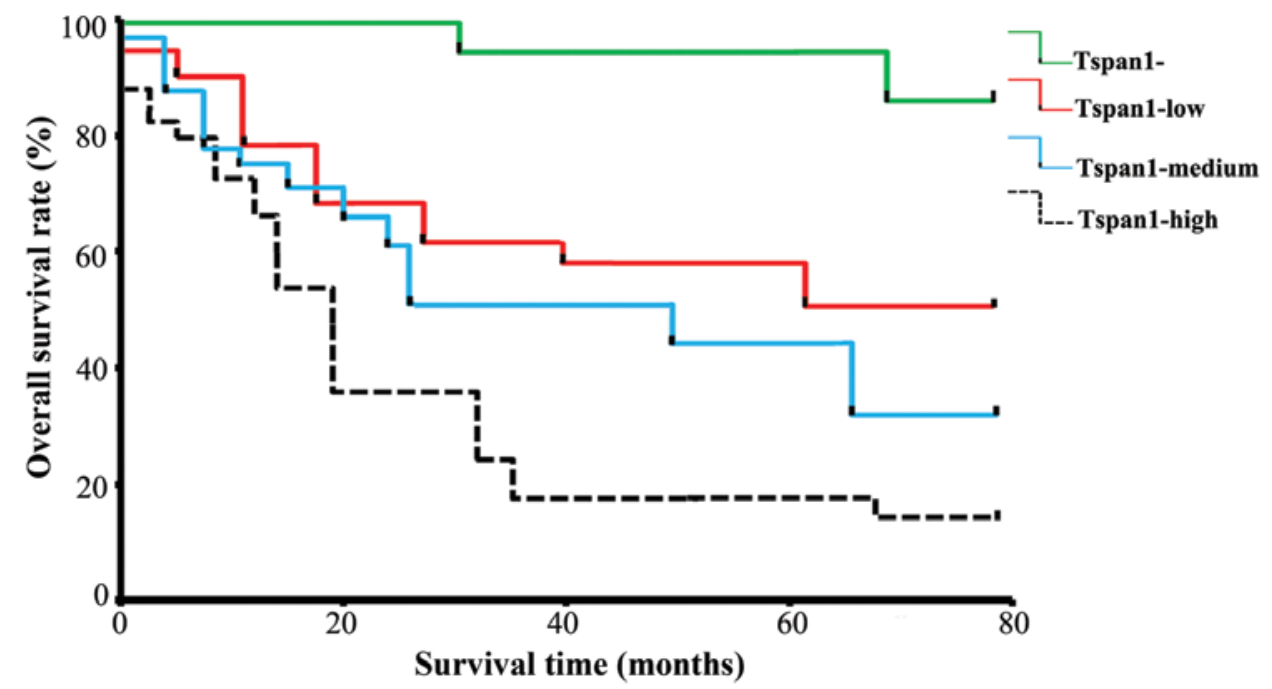

Figure 2. Overall 5-year survival curve of colorectal adenocarcinoma patients with Tspan1 negative and Tspan1 positive. PCC patients with high-positive Tspan1 protein expression had significantly worse total survival than those with low or medium positive expression. Survival rate in Tspan1 expression groups (Tspan1-low, -medium and -high) were obviously lower than that of negative (Tspan1') group $(\mathrm{P}<0.05$, respectively). Tspan1, tetraspanin 1; PCC, pancreatic cancer.

Tspan1 positive tumors. The 5 -year survival rate of pTNM stage I is $>95 \%$, while it is $<10 \%$ in patients with pTNM stage III-IV. The Kaplan-Meier estimates of overall survival rate were based on cell Tspan1 expression in the patients with 

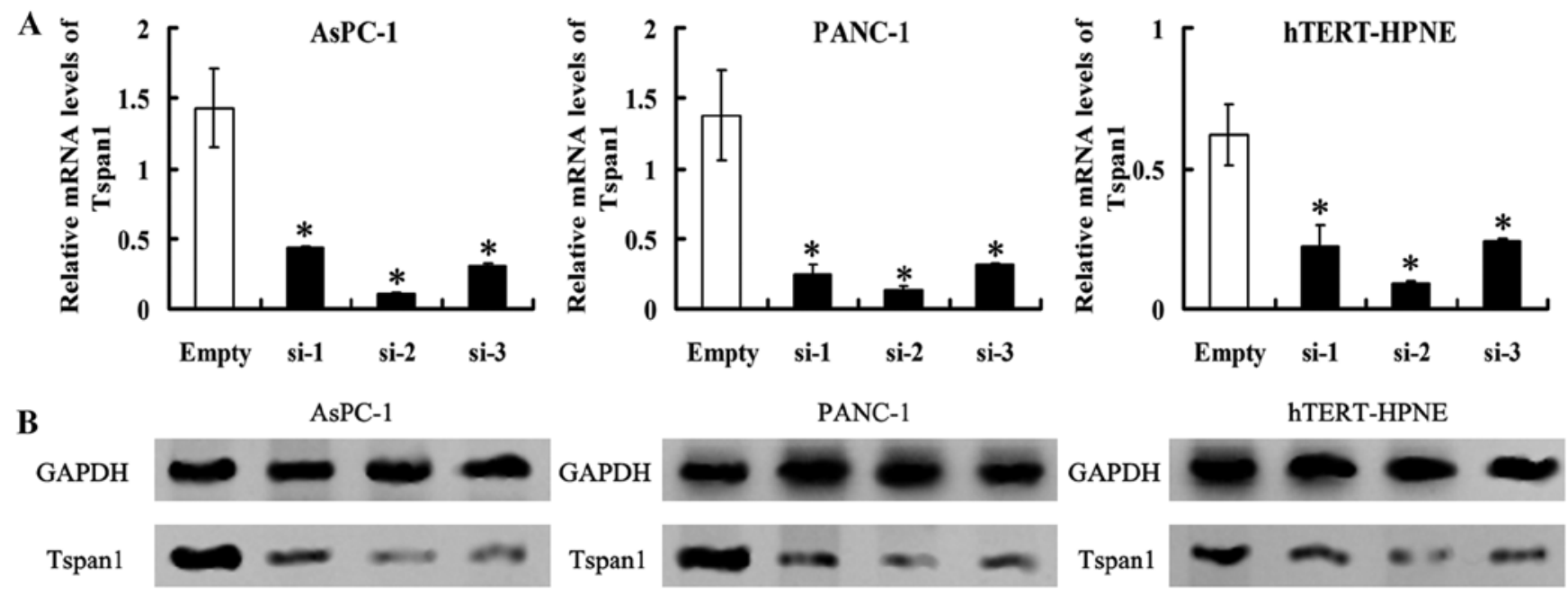

C
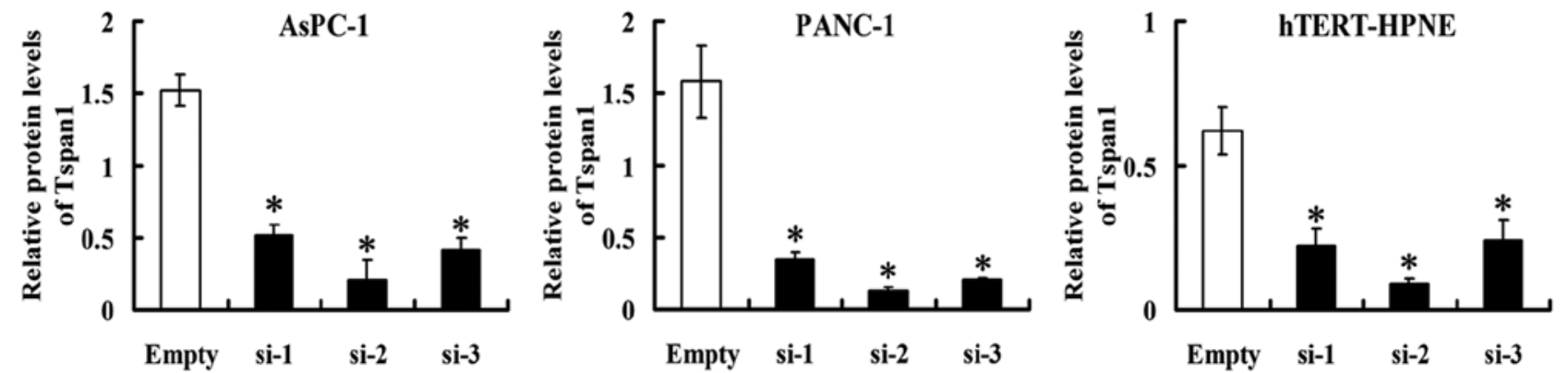

Figure 3. Expressions of Tspan1 in various cell lines after Tspan1-siRNA transfection. (A) Tspan1 mRNA levels detected by qRT-PCR were significantly lower in Tspan1-siRNA-expressed AsPC-1, PANC-1 and hTERT-HPNE cells than the matched control, respectively $(\mathrm{P}<0.05)$. (B) The protein levels of Tspan1 detected by western blot analysis. GAPDH was used as the control. (C) The quantity analysis of relative protein levels of Tspan1 after Tspan1-siRNA treatment in three PCC cell lines. Values plotted are means \pm SD $(n=3)$. "P<0.05 vs. Empty cell lines. Tspan1, tetraspanin 1; PCC, pancreatic cancer.

a follow-up period of 60 months (Fig. 2). In the entire cohort, the overall survival rate of patients with Tspan1-negative tumors was significantly higher than that in Tspan1-positive tumors (85.71 vs. $35.71 \%$; log-rank test: $\left.\chi^{2}=19.08, \mathrm{P}=0.0001\right)$. The relationship between Tspan1 expression pattern (IHC staining) and survival rate was also determined. Results revealed that Tspan1 higher-expression group had significantly shorter survival than the Tspan1 lower-expression group (Tspan1-medium vs. Tspan1-high, $\mathrm{P}<0.05$; Tspan1-negative vs. Tspan1-high, $\mathrm{P}<0.05$; Tspan1-medium vs. Tspan1-negative, $\mathrm{P}<0.05$; Fig. 2).

Interference expression of Tspanl by siRNA transfection. The PCC cell lines, AsPC-1 and PANC-1 cells, as well as the hTERT-HPNE cell stably transfected with Tspan1-siRNA-expressing vector (named as AsPC-1-Tspan1-si-1/2/3, PANC-1-Tspan1-si-1/2/3 and normalTspan1-si1/2/3, respectively). Control AsPC-1, PANC-1 and hTERT-HPNE cells were transfected with empty vectors. They were recorded as AsPC-1-Empty, PANC-1-Empty and normal-Empty, respectively. Tspan 1 mRNA levels detected by RT-PCR were significantly lower in Tspan1-siRNA expressed cells than the matched control $(\mathrm{P}<0.05$, Fig. 3). Western blot analysis found that the level of immunoreactive protein was significantly downregulated in Tspan1-siRNA-transfected cells relative to the controls cells $(\mathrm{P}<0.05$, Fig. 3$)$.
Stable expression of three Tspan1-siRNA (si-1, si-2 and si-3) in AsPC-1, PANC-1 and hTERT-HPNE cells resulted in $>60 \%$ decrease in Tspan 1 expression (Fig. 3). Considering the highest rates of inhibition of expression in Tspan1, AsPC-1-Tspan1-si-2 and PANC-1-Tspan1-si-2 were chosen as the target for further investigation.

Effects of Tspanl on PCC cells proliferation. We assessed the effect of Tspan1 expression silencing on the regulation of PCC cells viability. MTT assay showed that silencing of Tspan1 expression caused significant decrease in cell viability in AsPC-1 and PANC-1 cells, but not in hTERT-HPNE cells (Fig. 4).

Downregulation of Tspanl induces increased apoptosis of PCC cells. There was a significant increase in the apoptosis rate in Tspan1-siRNA-infected cells relative to empty-infected ones (Fig. 5). There were more apoptotic PCC cells in AsPC-1Tspan1-sh2 and PANC-1-Tspan1-sh1 groups, when compared with that of AsPC-1-Empty and PANC-1-Empty groups, respectively $(\mathrm{P}<0.05$, Fig. 5). However, Tspan1-siRNA showed no significant effects on the apoptosis of human normal hTERT-HPNE cells $(\mathrm{P}>0.05)$.

Effect of Tspanl knockdown on PDAC cell migration and invasion. Following knockdown, we compared the migration 

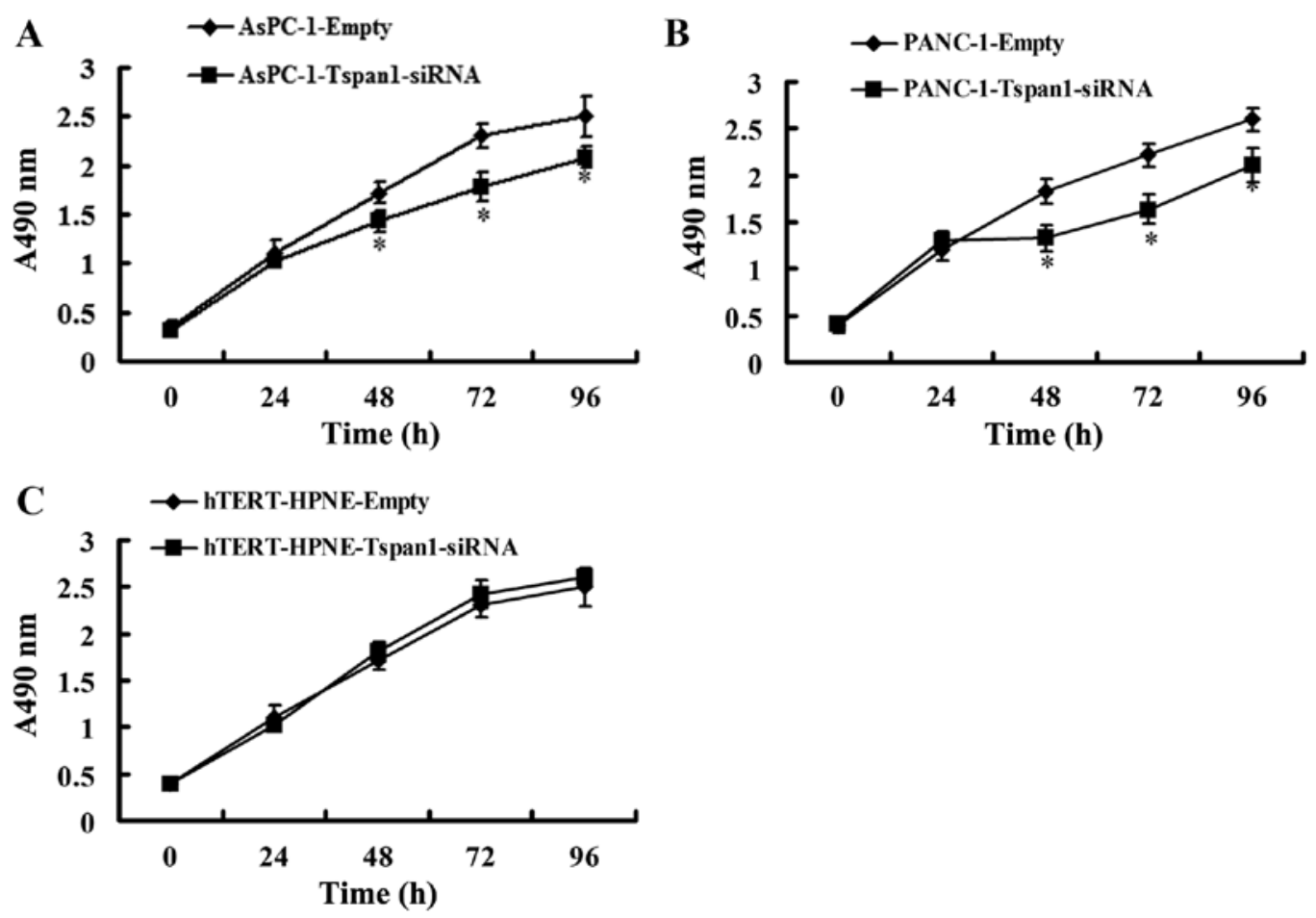

Figure 4. Effects of Tspan1 on PCC cell proliferation. MTT assay time-course for Empty and Tspan1-silence AsPC-1 cells (A), PANC-1 (B) and hTERT-HPNE cells (C). After Tspan1 downregulation, AsPC-1 and PANC-1 cells showed significant decrease in proliferation compared with that of control ones, respectively $(\mathrm{P}<0.05)$. There was no significant difference between hTERT-HPNE-Empty and hTERT-HPNE-Tspan1-siRNA group $(\mathrm{P}>0.05)$. Values plotted are means $\pm \mathrm{SD}(\mathrm{n}=3)$. ${ }^{*} \mathrm{P}<0.05$ vs. Empty cell lines. Tspan1, tetraspanin 1; PCC, pancreatic cancer.

of control non-transduced innocent cells (blank), non-targeting scrambled siRNA-transduced cells (negative control), as well as the Tspan1 knockdown cells transduced with Tspan1 targeting siRNA. The migration assay showed that the crystal violet stained cells significantly decreased in the Tspan1-siRNA-treated cells, compared with that of the matched WT and non-targeting siRNA control groups $(\mathrm{P}<0.01$, Fig. 6A). The Tspan 1 knockdown treatment significantly decreased the migration of the two cell types compared to the negative and blank control. Through the whole experimental duration, migration was not significantly different between AsPC-1 and PANC-1 blank cells and the negative control cells transduced with non-targeting scrambled siRNA, as shown in Fig. 6A.

There were significant reductions in the invasion of AsPC-1 and PANC-1 cells following Tspan1 knockdown, in comparison with that of the control cells, respectively ( $\mathrm{P}<0.005$, Fig. 6B). The invasion of control cells transduced with non-targeting siRNA, which had unchanged levels of Tspan1, was not significantly different from the non-transduced WT PCC cells.

\section{Discussion}

The present study focused on the possible roles of Tspan1 involved in the tumorigenic process of PCC. Our results revealed that Tspan1 was elevated in human PCC tissues and cell lines. The increased Tspan1 in PCC tissues was associated with the clinicopathological features and survival rate. Interference of Tspan1 expression by special siRNA introduction induced significant decline in proliferative capacity, increase in apoptosis and reduced migration and invasion of
AsPC-1 and PANC-1 cells, compared with that of hTERTHPNE cells. These data indicated that Tspan1 may be involved in the pathological changes and development of PCC.

Our results revealed that expression of Tspan1 in PCC cells displayed cytoplasmic patterns, which showed the distribution and functional sites of the Tspan1 molecule in cells (12). The molecule may accept extracellular signals when located on the membrane and carry out functions in the cytoplasm, like other tetraspanins such as CD9, CD82 and CD63 $(12,17,18)$. In the present study, we found that the expression of Tspan1 in PCC tissues was significantly higher than the normal adjacent tissues.

Tetraspanins, a large family of ubiquitously expressed membrane proteins, have been identified and implicated in the regulation of cell development, differentiation, proliferation, motility and tumor cell invasion (19-21). In many human cancers, tumor progression was found to be associated with an altered expression of tetraspanins (22). Tspan1, a new member of the tetraspanins group, was found to be elevated in some tumors (12,23-25). Recent studies also suggested Tspan1 gene may play a role in the proliferation of cancer cells and be associated with cancer cell motility, implying a function of the gene in the development of various cancer $(12,23,26)$. In this study, our results revealed that Tspan1 immunopositive staining was significantly correlated with the lymph node metastasis, pTNM stages and poor prognosis of PCC. Our data also showed that there was a significant correlation between the Tspan1 level and overall survival rate. Similarly, other reports show that there was a significant correlation between the Tspan 1 expression and overall survival rate, disease stages 

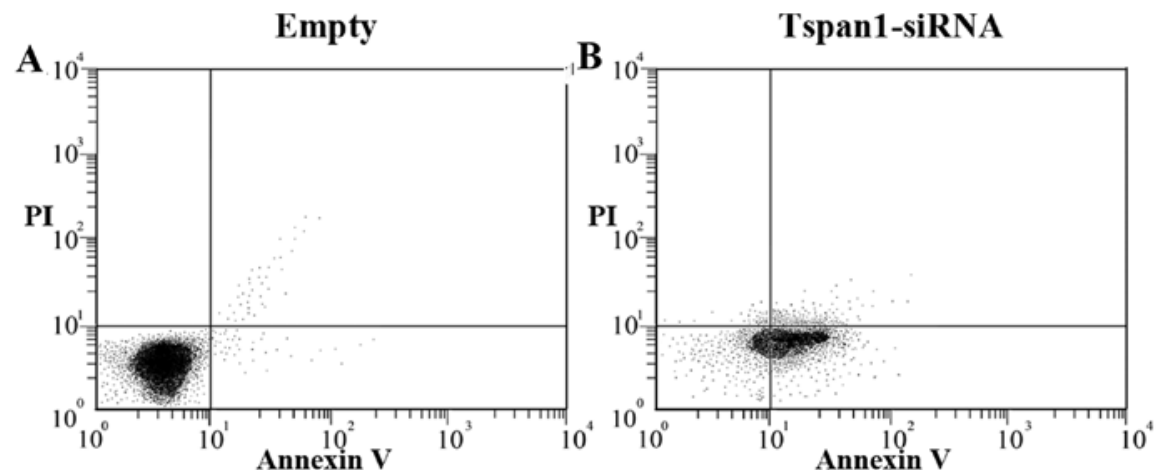

AsPC-1
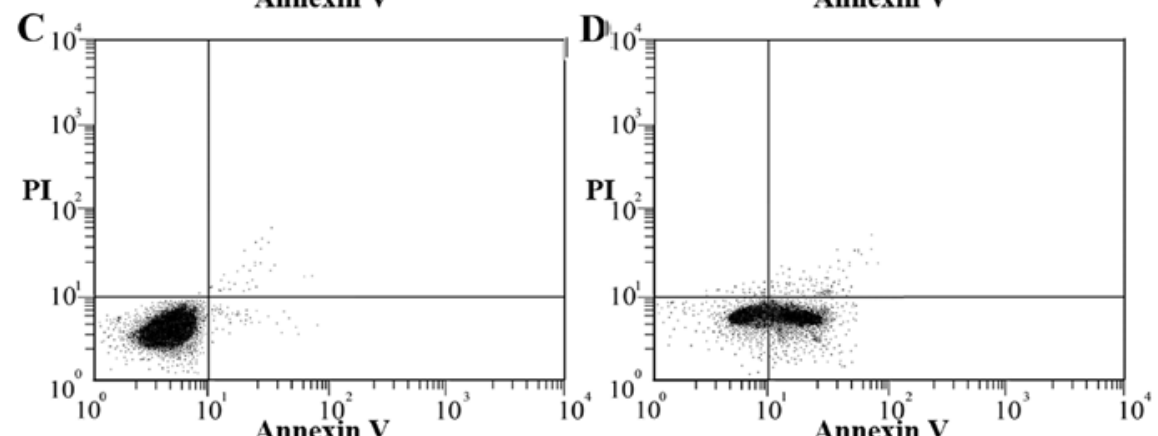

PANC-1
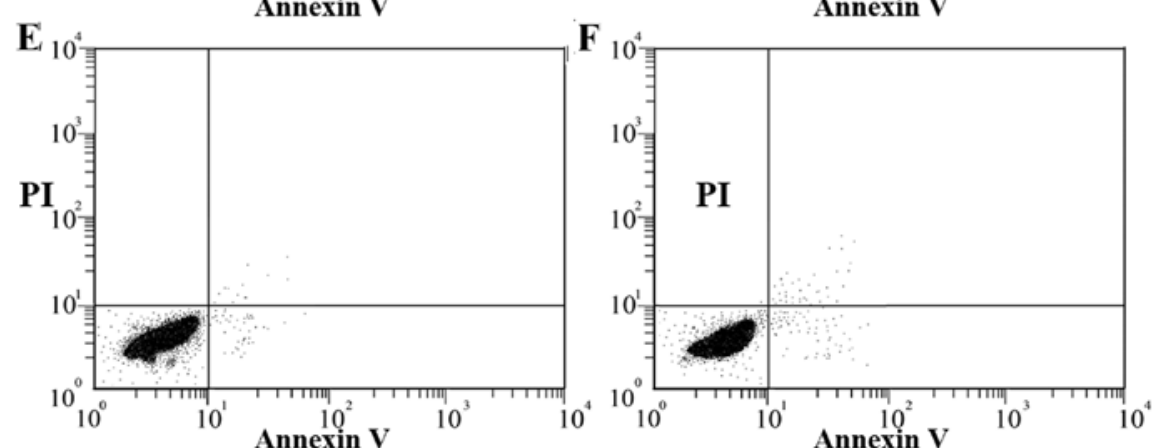

hTERT

-HPNE

G

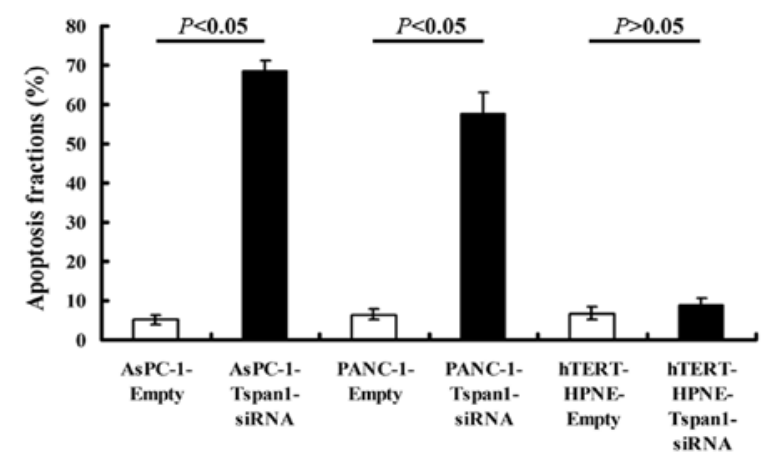

Figure 5. Tspan1 knockdown induces apoptosis in PCC cells. AsPC-1 cells, PANC-1 and hTERT-HPNE cells were transfected with Tspan1-siRNA or empty vector as control. (A-F) The influence of silencing Tspan1 on the apoptosis of AsPC-1 cells, PANC-1 and hTERT-HPNE was detected by FCM, respectively. (G) Quantification shows that the percentage of apoptotic cells in Tspan1-siRNA-transfected group is significantly higher compared to that in the Empty control group, except that in hTERT-HPNE cells. Values plotted are means \pm SD $(n=3)$. Tspan1, tetraspanin 1; PCC, pancreatic cancer; FCM, flow cytometry.

as well as the pathological features of other various tumors, such as colorectal cancer and cervical carcinoma $(13,23)$.

The present data also demonstrated that siRNA-mediated Tspan1 expression knockdown significantly inhibiting the growth, proliferation, migration and invasion of PCC cells in vitro, which was supported by earkier reports in different cancer cells $(10,12)$. We speculated that siRNA-mediated downregulation of Tspan1 inhibited the proliferation of PCC cells in vitro by inhibiting cell cycle progression from
G1 to S phase (26). Therefore, we postulated that: i), Tspan1 overexpression status may yield poor prognosis for PCC; and ii), Tspan1 may play a critical role in the progression of tumor growth and proliferation in human PCC.

In conclusion, our results show that Tspan1 was elevated in human PCC tissues and cell lines. Interference of Tspan1 expressions by siRNA introduction induced significant decline in proliferative capacity and increase in apoptosis of AsPC-1 and PANC-1 cells. This finding suggests that Tspan1 plays an 

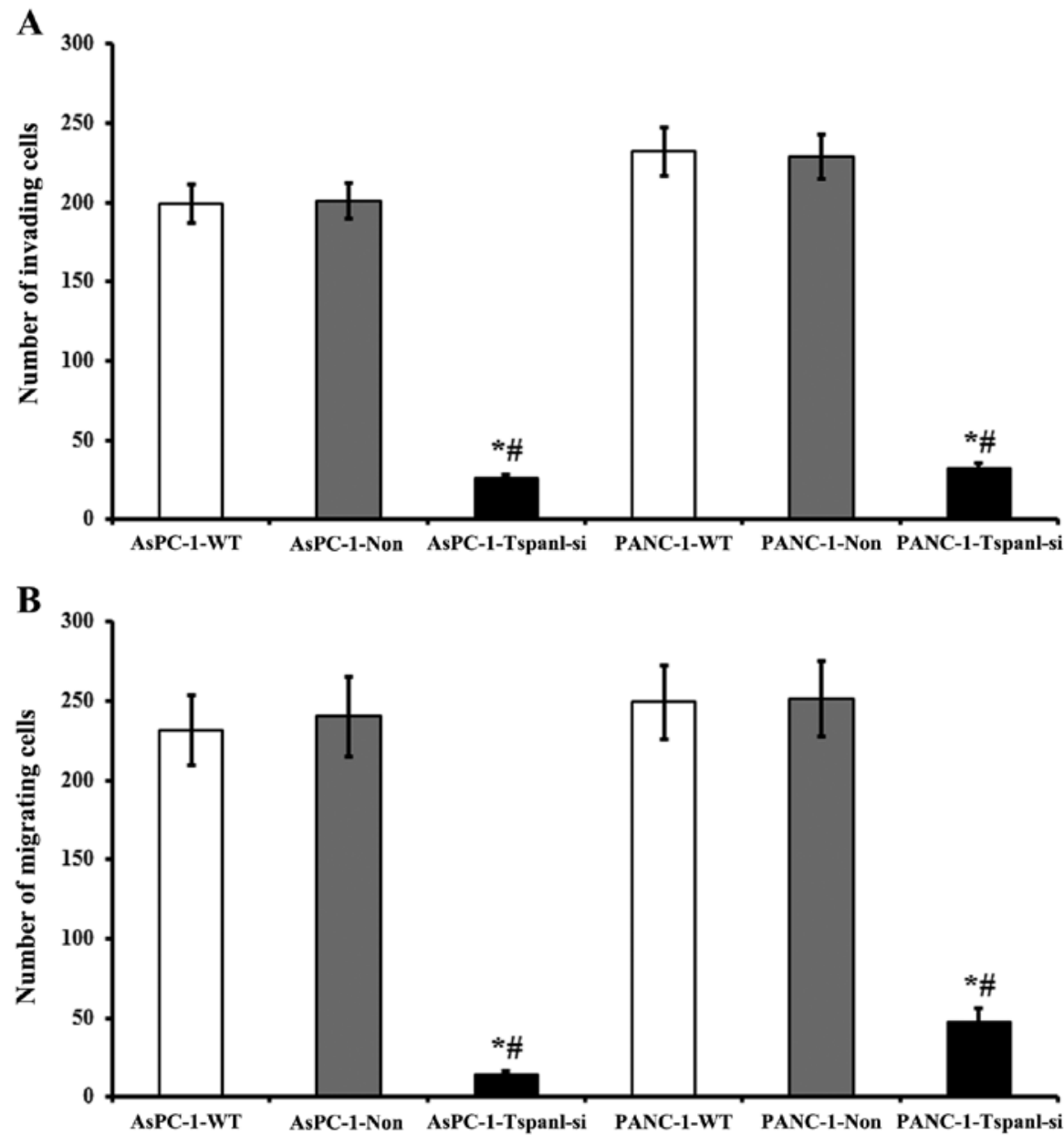

Figure 6. The effects of Tspan1 knockdown on the migration and invasion of AsPC-1/PANC-1 cells. (A) Migration was expressed as number of migrating cells (per 40x field). (B) Invasion was expressed as number of invading cells (per 40x field). Values plotted are means $\pm \mathrm{SD}$ ( $\mathrm{n}=3$ ). ${ }^{*} \mathrm{P}<0.05$ vs. AsPC-1/PANC-1-WT cell lines; ${ }^{\prime} \mathrm{P}<0.05$ vs. AsPC-1/PANC-1-Non cell lines. Tspan1, tetraspanin 1.

important role in PCC progression, and siRNA targeting of Tspan1 may be a potential therapeutic strategy for the treatment of PCC. Identifying the patients with high-risk PCC by Tspan1 expression detection would be of great benefit for improving treatment strategies.

\section{Acknowledgements}

We thank the Labreal Bioscience and Technology Ltd., Company, Kunming, China for valuable contribution to parts of the experimental design.

\section{References}

1. Bosetti C, Bertuccio P, Negri E, La Vecchia C, Zeegers MP and Boffetta P: Pancreatic cancer: Overview of descriptive epidemiology. Mol Carcinog 51: 3-13, 2012.

2. Vincent A, Herman J, Schulick R, Hruban RH and Goggins M: Pancreatic cancer. Lancet 378: 607-620, 2011.

3. Loos M, Kleeff J, Friess H and Büchler MW: Surgical treatment of pancreatic cancer. Ann NY Acad Sci 1138: 169-180, 2008.

4. Veenbergen $S$ and van Spriel AB: Tetraspanins in the immune response against cancer. Immunol Lett 138: 129-136, 2011.

5. Yamamoto Y, Grubisic K and Oelgeschläger M: Xenopus tetraspanin-1 regulates gastrulation movements and neural differentiation in the early Xenopus embryo. Differentiation 75 $235-245,2007$
6. Huang S, Yuan S, Dong M, Su J, Yu C, Shen Y, Xie X, Yu Y, $\mathrm{Yu} X$, Chen $\mathrm{S}$, et al: The phylogenetic analysis of tetraspanins projects the evolution of cell-cell interactions from unicellular to multicellular organisms. Genomics 86: 674-684, 2005.

7. André M, Chambrion C, Charrin S, Soave S, Chaker J, Boucheix C, Rubinstein E and Le Naour F: In situ chemical cross-linking on living cells reveals CD9P-1 cis-oligomer at cell surface. J Proteomics 73: 93-102, 2009.

8. Hölters S, Anacker J, Jansen L, Beer-Grondke K, Dürst M and Rubio I: Tetraspanin 1 promotes invasiveness of cervical cancer cells. Int J Oncol 43: 503-512, 2013.

9. Chen Y, Peng W, Lu Y, Chen J, Zhu YY and Xi T: miR-200a enhances the migrations of A549 and SK-MES-1 cells by regulating the expression of TSPAN1. J Biosci 38: 523-532, 2013.

10. Chen L, Yuan D, Zhao R, Li H and Zhu J: Suppression of TSPAN1 by RNA interference inhibits proliferation and invasion of colon cancer cells in vitro. Tumori 96: 744-750, 2010.

11. Desouki MM, Liao S, Huang H, Conroy J, Nowak NJ, Shepherd L, Gaile DP and Geradts J: Identification of metastasis-associated breast cancer genes using a high-resolution whole genome profiling approach. J Cancer Res Clin Oncol 137: 795-809, 2011.

12. Chen L, Zhu Y, Li H, Wang GL, Wu YY, Lu YX, Qin J, Tuo J, Wang JL and Zhu J: Knockdown of TSPAN1 by RNA silencing and antisense technique inhibits proliferation and infiltration of human skin squamous carcinoma cells. Tumori 96: 289-295, 2010.

13. Chen L, Zhu YY, Zhang XJ, Wang GL, Li XY, He S, Zhang JB and Zhu JW: TSPAN1 protein expression: A significant prognostic indicator for patients with colorectal adenocarcinoma. World J Gastroenterol 15: 2270-2276, 2009.

14. Jiang SH, He P, Ma MZ, Wang Y, Li RK, Fang F, Fu Y, Tian GA, Qin WX and Zhang ZG: PNMA1 promotes cell growth in human pancreatic ductal adenocarcinoma. Int J Clin Exp Pathol 7: 3827-3835, 2014. 
15. Sun GG, Wei CD, Jing SW and Hu WN: Interactions between filamin A and MMP-9 regulate proliferation and invasion in renal cell carcinoma. Asian Pac J Cancer Prev 15: 3789-3795, 2014.

16. Kramer N, Walzl A, Unger C, Rosner M, Krupitza G, Hengstschläger $\mathrm{M}$ and Dolznig $\mathrm{H}$ : In vitro cell migration and invasion assays. Mutat Res 752: 10-24, 2013.

17. Hemler ME: Tetraspanin functions and associated microdomains. Nat Rev Mol Cell Biol 6: 801-811, 2005.

18. Zöller M: Tetraspanins: Push and pull in suppressing and promoting metastasis. Nat Rev Cancer 9: 40-55, 2009.

19. Mazzocca A, Carloni V, Sciammetta S, Cordella C, Pantaleo P, Caldini A, Gentilini P and Pinzani M: Expression of transmembrane 4 superfamily (TM4SF) proteins and their role in hepatic stellate cell motility and wound healing migration. J Hepatol 37: 322-330, 2002.

20. Furuya M, Kato H, Nishimura N, Ishiwata I, Ikeda H, Ito R, Yoshiki $\mathrm{T}$ and Ishikura H: Down-regulation of CD9 in human ovarian carcinoma cell might contribute to peritoneal dissemination: Morphologic alteration and reduced expression of beta1 integrin subsets. Cancer Res 65: 2617-2625, 2005.

21. Chen Z, Mustafa T, Trojanowicz B, Brauckhoff M, Gimm O, Schmutzler C, Köhrle J, Holzhausen HJ, Kehlen A, Klonisch T, et al: CD82, and CD63 in thyroid cancer. Int J Mol Med 14: $517-527,2004$
22. Klosek SK, Nakashiro K, Hara S, Goda H, Hasegawa H and Hamakawa H: CD151 regulates HGF-stimulated morphogenesis of human breast cancer cells. Biochem Biophys Res Commun 379: 1097-1100, 2009.

23. Wollscheid V, Kühne-Heid R, Stein I, Jansen L, Köllner S, Schneider A and Dürst M: Identification of a new proliferation-associated protein NET-1/C4.8 characteristic for a subset of high-grade cervical intraepithelial neoplasia and cervical carcinomas. Int J Cancer 99: 771-775, 2002.

24. Chen L, Wang Z, Zhan X, Li DC, Zhu YY and Zhu J: Association of NET-1 gene expression with human hepatocellular carcinoma. Int J Surg Pathol 15: 346-353, 2007.

25. Scholz CJ, Kurzeder C, Koretz K, Windisch J, Kreienberg R, Sauer G and Deissler H: Tspan-1 is a tetraspanin preferentially expressed by mucinous and endometrioid subtypes of human ovarian carcinomas. Cancer Lett 275: 198-203, 2009.

26. Leyden J, Murray D, Moss A, Arumuguma M, Doyle E, McEntee G, O'Keane C, Doran P and MacMathuna P: Net1 and Myeov: Computationally identified mediators of gastric cancer. Br J Cancer 94: 1204-1212, 2006. 